\title{
PROPERTIES OF ALMOST CONTINUOUS FUNCTIONS
}

\author{
PAUL E. LONG AND EARL E. MCGEHEE, JR.
}

Introduction. Professor T. Husain [2] has defined the concept of an almost continuous function from one topological space into another and investigated some of their properties. His definition is as follows:

Definition 1. The function $f: X \rightarrow Y$ is almost continuous at $x_{0} \in X$ if and only if for each open $V \subset Y$ containing $f\left(x_{0}\right), \mathrm{Cl}\left(f^{-1}(V)\right)$ is a neighborhood of $x_{0}$. If $f$ is almost continuous at each point of $X$, then $f$ is called almost continuous.

We prove several results concerning almost continuous functions and point out that the hypotheses on two of Husain's theorems may be weakened considerably. Functions having closed graphs also play an important role in our investigation.

We note that Stallings [6] has also defined a type of function which he calls almost continuous. His definition is given next.

Definition 2. The function $f: X \rightarrow Y$ is almost continuous if and only if given any open set $W$ containing the graph of $f$, there exists a continuous $g: X \rightarrow Y$ such that the graph of $g$ is a subset of $W$.

Of course, any continuous function satisfies both definitions. However, the following examples show that neither of these two almost continuous functions, in general, imply the other. Example 1 gives a function $f$ which is almost continuous in the sense of Husain but is not almost continuous in the sense of Stallings. Furthermore, $f$ is not a connected function (i.e., $f$ does not preserve connected subsets of $X$ ) and is not a connectivity function (i.e., the graph map $g(x)=(x, f(x))$ from $X$ into $X \times Y$ is not a connected function). Example 2 gives a function which is almost continuous in the sense of Stallings, is connected, is a connectivity function but is not almost continuous in the sense of Husain.

EXAMPLE 1. Let $R$ represent the reals with standard topology. Let $f: R \rightarrow R$ be given by $f(x)=x$ if $x$ is rational and $f(x)=-x$ if $x$ is irrational.

Example 2. With $R$ as in Example 1, define $f: R \rightarrow R$ as $f(x)$ $=\sin (1 / x)$ if $x \neq 0$, and $f(0)=0$.

When dealing with almost continuous functions throughout the remainder of this paper, we will be referring only to those of the type described by Husain. Hereafter, we abbreviate "almost continuous" with a.c. The notation $\mathrm{Cl}(A)$ will denote the closure of the set $A$ and

Received by the editors March 28, 1969. 
the symbol $G(f)$ will denote the graph of the function $f$. The symbol $R$ will denote the reals with standard topology. No separation axioms are assumed on the spaces unless explicitly stated.

Results. Our first theorems concern sufficient and necessary conditions that a function be a.c.

TheOREM 1. The function $f: X \rightarrow Y$ is a.c. at $x_{0} \in X$ if and only if for each open $V \subset Y$ containing $f\left(x_{0}\right)$, there exists an open set $U \subset X$ containing $x_{0}$ such that $f^{-1}(V)$ is dense in $U$.

Proof. The proof is a direct consequence of Definition 1.

Lemma. Let $f: X \rightarrow Y$ be a.c. at $x_{0} \in X$ where $X$ is $T_{1}$ and $x_{0} \in X$ is a limit point of $X$. Then for every pair of open sets $U \subset X$ and $V \subset Y$ containing $x_{0}$ and $f\left(x_{0}\right)$, respectively, there exists an $x \in U \backslash\left\{x_{0}\right\}$ such that $f(x) \in V$.

Proof. Assume the conclusion false. Then there exist open sets $U_{1}$ and $V_{1}$ containing $x_{0}$ and $f\left(x_{0}\right)$, respectively, such that $U_{1} \cap f^{-1}\left(V_{1}\right)$ $=\left\{x_{0}\right\}$. Thus no point of $U_{1}$ is a limit point of $f^{-1}\left(V_{1}\right)$ in the $T_{1}$ space $X$. Therefore $U_{1} \cap \mathrm{Cl}\left(f^{-1}\left(V_{1}\right)\right)=\left\{x_{0}\right\}$ which contradicts the hypothesis that $f$ is a.c. at $x_{0}$. This proves the lemma.

Theorem 2. Let $f: X \rightarrow Y$ be a.c. at $x_{0} \in X$ where $X$ is $T_{1}$ and both $X$ and $Y$ are first countable. If $x_{0}$ is a limit point of $X$, there exists a sequence $\left\{x_{n}\right\}$ of distinct points in $X$ converging to $x_{0}$ such that $\left\{f\left(x_{n}\right)\right\}$ converges to $f\left(x_{0}\right)$.

Proof. Let $\left\{U_{n}\right\}$ and $\left\{V_{n}\right\}$ be countable descending bases at the points $x_{0}$ and $f\left(x_{0}\right)$, respectively. According to the lemma, for the two open sets $U_{1}$ and $V_{1}$, there is a point $x_{1} \in U_{1} \backslash\left\{x_{0}\right\}$ such that $f\left(x_{1}\right) \in V_{1}$. Now considering the open sets $U_{2}$ and $V_{2}, U_{2} \backslash\left\{x_{1}\right\}$ is an open set containing $x_{0}$ in the $T_{1}$ space $X$ and again by the lemma, there exists a point $x_{2} \in U_{2} \backslash\left\{x_{0}, x_{1}\right\}$ such that $f\left(x_{2}\right) \in V_{2}$. Inductively, for the open sets $U_{k}$ and $V_{k}, U_{k} \backslash\left\{x_{1}, x_{2}, \cdots, x_{k-1}\right\}$ is open, contains $x_{0}$ and hence there exists a point $x_{k} \in U_{k} \backslash\left\{x_{0}, x_{1}, \cdots, x_{k-1}\right\}$ such that $f\left(x_{k}\right) \in V_{k}$. The existence of $x_{k}$ is guaranteed by the fact that an open set containing the limit point $x_{0}$ of the $T_{1}$ space $X$ contains infinitely many points of $X$. Thus we have constructed a sequence $\left\{x_{n}\right\}$ of distinct points in $X$ converging to $x_{0}$ such that $f\left(x_{n}\right) \in V_{n}$ for each positive integer $n$.

COROLLARY. Under the hypothesis of Theorem 2, where in addition $X$ and $Y$ are $T_{2}$, the point $x_{0}$ is not a removable discontinuity of $f$. 
TheOREM 3. Let $f: X \rightarrow Y$ be any function and let $D$ be a dense subset of $X$. If $f \mid D$ is continuous, then $f$ is a.c. at each point of $D$.

Proof. Let $x_{0} \in D$ and $V$ any open set containing $f\left(x_{0}\right)$. Since $f \mid D$ is continuous, there exists an open set $U \subset X$ containing $x_{0}$ such that $(D \cap U) \subset(f \mid D)^{-1}(V)=D \cap f^{-1}(V)$ and hence $(D \cap U) \subset f^{-1}(V)$. Since $D$ is dense, $U \subset \mathrm{Cl}(D \cap U)$ by Exercise $\mathrm{G}$ of $[4$, p. 57], and hence $U \subset \mathrm{Cl}\left(f^{-1}(V)\right)$. Thus $f$ is a.c. at $x_{0}$.

The following example shows that restrictions of a.c. functions to arbitrary subsets of the domain may not be a.c.

Example 3. Consider the a.c. function $f: R \rightarrow R$ given by $f(x)=1$ if $x$ is rational and $f(x)=-1$ if $x$ is irrational. Let $M$ be the set consisting of all nonnegative rationals together with all negative irrationals. Then $f \mid M$ is not a.c. at $x=0$.

We do make the following observations, however.

Theorem 4. If $f: X \rightarrow Y$ is a.c. and $U$ is an open subset of $X$, then $f \mid U$ is a.c.

The following example, due to Kuratowski and discussed briefly in [5], describes a function which is connected and a.c. but not continuous.

EXAmple 4. Let $f$ map $I=[0,1]$ into $I$ defined as follows: $f(x)$ $=\lim \sup \left(a_{1}+a_{2}+\cdots+a_{n}\right) / n$ for each $x \in I$ where the $a_{i}$ are given by the unique nonterminating binary expansion of the number $x$ $=\left(0 . a_{1} a_{2} \cdots\right)$.

Our next theorem gives conditions when a connected function is also a.c.

Definition 3. The function $f: X \rightarrow Y$ is called finitely closed at $x_{0} \in X$ if and only if for each open set $V \subset Y$ containing $f\left(x_{0}\right)$ there exists an open set $V_{1}$ such that $f\left(x_{0}\right) \in V_{1} \subset V$ and $X \backslash \mathrm{Cl}\left(f^{-1}\left(V_{1}\right)\right)$ consists of finitely many components.

Theorem 5. Let $f: X \rightarrow Y$ be connected where $Y$ is regular. If $f$ is finitely closed at $x_{0} \in X$, then $f$ is a.c. at $x_{0}$.

Proof. If $f$ is not a.c. at $x_{0} \in X$, there exists an open set $V \subset Y$ containing $f\left(x_{0}\right)$ such that $x_{0}$ is not interior to $\mathrm{Cl}\left(f^{-1}(V)\right)$. Thus $x_{0}$ is a limit point of $X \backslash \mathrm{Cl}\left(f^{-1}(V)\right)$. Since $f$ is finitely closed at $x_{0}$ there exists an open set $V_{1}$ containing $f\left(x_{0}\right)$ where $V_{1} \subset V$ and such that $X \backslash \mathrm{Cl}\left(f^{-1}\left(V_{1}\right)\right)$ consists of finitely many components $C_{1}, C_{2}, C_{3}, \cdots$, $C_{n}$. Because $\mathrm{Cl}\left(f^{-1}\left(V_{1}\right)\right) \subset \mathrm{Cl}\left(f^{-1}(V)\right), x_{0}$ is not interior to $\mathrm{Cl}\left(f^{-1}\left(V_{1}\right)\right)$ so that $x_{0}$ is a limit point of $X \backslash \mathrm{Cl}\left(f^{-1}\left(V_{1}\right)\right)$ and hence a limit point of some $C_{k}, 1 \leqq k \leqq n$. Thus $C_{k} \cup\left\{x_{0}\right\}$ is connected but $f\left(C_{k} \cup\left\{x_{0}\right\}\right)$ $=f\left(C_{k}\right) \cup\left\{f\left(x_{0}\right)\right\}$ is not connected in the regular space $Y$ because 
$f\left(x_{0}\right) \in V_{1}$ while $f\left(C_{k}\right) \subset Y \backslash V_{1}$. This contradiction to the connected hypothesis of $f$ means $f$ is a.c. at $x_{0}$.

For any function $f: X \rightarrow Y, \mathrm{G}(f)$ is a closed subset of $X \times Y$ if and only if for each $x_{0} \in X$ and $y \neq f\left(x_{0}\right)$, there exist open sets $U$ and $V$ containing $x_{0}$ and $y$, respectively, such that $f(U) \cap V=\varnothing$. Fuller $[1$, Theorem 3.6] has shown that if $f: X \rightarrow Y$ is any function having closed graph, where $X$ and $Y$ are arbitrary spaces and $M \subset Y$ is compact, then $f^{-1}(M)$ is a closed subset of $X$. From this it easily follows that for functions $f: X \rightarrow Y$ having closed graphs and $Y$ compact, then $f$ is continuous. Theorem 1 of [2] states that an a.c. function $f: X \rightarrow Y$ which is completely closed where $Y$ is compact and $T_{2}$ is continuous. Our preceding remarks show these hypotheses may be substantially weakened. The same holds true for Theorem 3 of [2]. Next we prove a converse to Fuller's theorem and then relate a.c. functions, functions having closed graphs and continuous functions.

Theorem 6. Let $f: X \rightarrow Y$ be any function where $Y$ is locally compact and either a regular $T_{1}$ space or a Hausdorff space. If for each compact $K \subset Y, f^{-1}(K)$ is closed, then $G(f)$ is closed.

Proof. Let $x_{0} \in X$ and $y \neq f\left(x_{0}\right)$. Since $Y$ is either $T_{1}$ or $T_{2}$, there exists a neighborhood $V$ containing $y$ such that $f\left(x_{0}\right) \notin V$. By Theorem 17 of $[4$, p. 146], the collection of closed compact neighborhoods of $y$ form a base for its neighborhood system. Thus there exists a closed compact neighborhood $V_{1} \subset V$ containing $y$ and from the hypothesis it follows that $f^{-1}\left(V_{1}\right)$ is closed in $X$. Consequently, $X \backslash f^{-1}\left(V_{1}\right)=U$ is open and $f(U) \cap V_{\mathbf{1}}=\varnothing$ which implies $G(f)$ is closed.

Theorem 7. Let $f: X \rightarrow Y$ be a.c. where $f$ is injective and has closed graph. Then $X$ is $T_{2}$.

Proof. Let $x, w \in X$ be distinct points. Then $f(x) \neq f(w)$ and since $G(f)$ is closed, there exist open sets $U$ and $V$ containing $x$ and $f(w)$, respectively, such that $f(U) \cap V=\varnothing$. Thus $f^{-1}(V) \subset X \backslash U$ and $\mathrm{Cl}\left(f^{-1}(V)\right)$ is a neighborhood of $w$ because $f$ is a.c. Since $X \backslash U$ is closed, $\mathrm{Cl}\left(f^{-1}(V)\right) \subset X \backslash U$. Therefore there exist disjoint open sets in $X$ containing $x$ and $w$, respectively, implying $X$ is $T_{2}$.

THEOREM 8. Let $f: X \rightarrow Y$ be a.c. where $Y$ is locally connected and $T_{2}$. If both $f$ and $f^{-1}$ preserve connected sets, then $G(f)$ is closed.

Proof. Let $x_{0} \in X$ and $y \neq f\left(x_{0}\right)$ belong to $Y$. Then there exist open disjoint sets $U$ and $\mathrm{V}$ containing $y$ and $f\left(x_{0}\right)$, respectively, since $Y$ is $T_{2}$. By local connectedness of $Y$, there is a connected open set $W \subset V$ containing $f\left(x_{0}\right)$; hence $U \cap W=\varnothing$ so that $f^{-1}(U) \cap f^{-1}(W)=\varnothing$. If 
any $x \in f^{-1}(U)$ is a limit point of the connected set $f^{-1}(W)$, then $\{x\} \cup f^{-1}(W)$ is a connected set but $f\left(\{x\} \cup f^{-1}(W)\right)$ is not connected since it has points in each of two disjoint open sets $U$ and $W$. This contradicts $f$ being connected. Therefore $\mathrm{Cl}\left(f^{-1}(W)\right) \cap f^{-1}(U)=\varnothing$. Since $f$ is a.c., there is an open set $T \subset \mathrm{Cl}\left(f^{-1}(W)\right)$ containing $x_{0}$ which means $f(T) \cap U=\varnothing$. Consequently, $G(f)$ is closed.

THeOREM 9. Let $f: X \rightarrow Y$ be a.c. where $Y$ is locally compact. If $Y$ is either regular or Hausdorff and $G(f)$ is closed, then $f$ is continuous.

Proof. Let $x_{0} \in X$ and $V$ a neighborhood of $f\left(x_{0}\right)$. By our hypothesis, Theorem 17 of $[4$, p. 146] implies the collection of closed compact neighborhoods of $f\left(x_{0}\right)$ is a base for the neighborhood system of $f\left(x_{0}\right)$. Thus there exists a compact neighborhood $W$ of $f\left(x_{0}\right)$ such that $W \subset V$. Since $G(f)$ is closed, $f^{-1}(W)$ is closed by Theorem 3.6 [1] so that $\mathrm{Cl}\left(f^{-1}(W)\right)=f^{-1}(W)$. But $\mathrm{Cl}\left(f^{-1}(W)\right)$ is a neighborhood of $x_{0}$ because $f$ is a.c. which implies $f^{-1}(V)$ is a neighborhood of $x_{0}$. Thus $f$ is continuous at $x_{0}$.

Corollary. Let $f: R \rightarrow R$ be a.c. If $G(f)$ is closed, then $f$ is continuous.

Theorem 10. Let $f: X \rightarrow Y$ be a.c. where $Y$ is a regular, locally connected space. If $f$ has the property that $\mathrm{Cl}\left(f^{-1}(C)\right) \subset f^{-1}(\mathrm{Cl}(C))$ for every connected subset $C \subset Y$, then $f$ is continuous.

Proof. Let $x_{0} \in X$ and let $V$ be any open set containing $f\left(x_{0}\right)$. Since $Y$ is regular and locally connected, there exists a connected open set $W \subset V$ containing $f\left(x_{0}\right)$ such that $\mathrm{Cl}(W) \subset V$. Thus $f^{-1}(\mathrm{Cl}(W))$ $\subset f^{-1}(V)$. By hypothesis, $\mathrm{Cl}\left(f^{-1}(W)\right) \subset f^{-1}(\mathrm{Cl}(W))$ since $W$ is connected, and therefore $\operatorname{Cl}\left(f^{-1}(W)\right) \subset f^{-1}(V)$. But $f$ is a.c. so that $\mathrm{Cl}\left(f^{-1}(W)\right)$ is a neighborhood of $x_{0}$ and hence $f^{-1}(V)$ is a neighborhood of $x_{0}$. Consequently, $f$ is continuous at $x_{0}$.

Theorem 11. Let $f: X \rightarrow Y$ be a.c. where $X$ is $T_{2}$ and $Y$ is a regular, locally connected $T_{2}$ space. If $f$ is connected and $f^{-1}(C)$ is connected for every connected subset $C \subset Y$, then $f$ is continuous.

Proof. Let $C$ be any connected subset of $Y$ and let $K=f^{-1}(C)$. The set $K$ is connected since $f^{-1}$ preserves connected sets and furthermore $f(\mathrm{Cl}(K)) \subset \mathrm{Cl}(f(K))$ by Theorem 3.3 of [5] because $f$ is a connected function. Thus $f(\mathrm{Cl}(K)) \subset \mathrm{Cl}(f(K)) \subset \mathrm{Cl}(C)$, which implies $f^{-1}(f(\mathrm{Cl}(K))) \subset f^{-1}(\mathrm{Cl}(C))$. Since $\mathrm{Cl}(K) \subset f^{-1}(f(\mathrm{Cl}(K)))$, it follows that $\mathrm{Cl}(K) \subset f^{-1}(\mathrm{Cl}(C))$. Using the fact that $K=f^{-1}(C)$, we then have $\mathrm{Cl}\left(f^{-1}(C)\right) \subset f^{-1}(\mathrm{Cl}(C))$. Applying Theorem 10 to this last subset implication, it follows that $f$ is continuous. 
Jones [3] has defined a semiconnected map $f: X \rightarrow Y$ as one in which $f^{-1}$ preserves closed connected subsets of $Y$. The following theorem states conditions under which a function that is both semiconnected and a.c. is continuous.

Theorem 12. Let $f: X \rightarrow Y$ be a.c. where $Y$ is locally connected and regular. If $f$ is semiconnected, then $f$ is continuous.

Proof. Let $x_{0} \in X$ and $V$ any open set containing $f\left(x_{0}\right)$. By the hypothesis on $Y$, there exists a connected open set $W$ containing $f\left(x_{0}\right)$ such that $\mathrm{Cl}(W) \subset V$. Therefore $f^{-1}(W) \subset f^{-1}(\mathrm{Cl}(W)) \subset f^{-1}(V)$ and since $f^{-1}(\mathrm{Cl}(W))$ is closed due to semiconnectedness of $f, \mathrm{Cl}\left(f^{-1}(W)\right)$ $\subset f^{-1}(\mathrm{Cl}(W)) \subset f^{-1}(V)$. Since $f$ is a.c., there is a neighborhood $T$ $\subset \mathrm{Cl}\left(f^{-1}(W)\right)$ containing $x_{0}$. Thus $f(T) \subset V$ and $f$ is continuous at $x_{0}$.

Theorem 13. Let $f: R \rightarrow Y$ be a.c. where $Y$ is locally connected. If $f^{-1}(C)$ is connected for every connected subset $C$ of $Y$, then $f$ is continuous.

Proof. Let $x_{0} \in R$ and let $V$ be any open set containing $f\left(x_{0}\right)$. Since $Y$ is locally connected, $V$ may be assumed connected. By the hypothesis on $f, f^{-1}(V)$ is a connected subset of $R$ and is therefore an interval containing $x_{0}$. Consequently, $\mathrm{Cl}\left(f^{-1}(V)\right)$ is an interval and since $f$ is a.c., $\mathrm{Cl}\left(f^{-1}(V)\right)$ is a neighborhood of $x_{0}$ implying $x_{0}$ is an interior point of $f^{-1}(V)$. Thus $f$ is continuous at $x_{0}$.

CoROllaRy. Let $f: R \rightarrow R$ be a.c. If $f^{-1}(C)$ is connected for every connected subset $C$ of $R$, then $f$ is continuous.

It would be interesting to know under what conditions an a.c. function of the type described by Husain is also one of the type described by Stallings and conversely. Also, what are the relations between Husain a.c. functions, connectivity functions and connected functions?

\section{REFERENCES}

1. R. V. Fuller, Relations among continuous and various non-continuous functions, Pacific J. Math. 25 (1968), 495-509. MR 37 \#3536.

2. T. Husain, Almost continuous mappings, Prace. Mat. 10 (1966), 1-7. MR 36 \#3322.

3. John Jones, Jr., On semi-connected mappings of topological spaces, Proc. Amer. Math. Soc. 19 (1968), 174-175. MR 36 \#5907.

4. John L. Kelley, General topology, Van Nostrand, Princeton, N. J., 1955. MR 16, 1136.

5. W. J. Pervin and Norman Levine, Connected mappings of Hausdorff spaces, Proc. Amer. Math. Soc. 9 (1958), 488-495. MR 20 \#1970.

6. J. Stallings, Fixed point theorems for connectivity maps, Fund. Math. 47 (1959), 249-263. MR 22 \#8485.

University of ARKansas 